Classification

Physics Abstracts

$34.50 \mathrm{H}-35.10 \mathrm{D}-35.80$

\title{
Tensor polarizability measurement by a quantum beat method
}

\author{
Y. Ouerdane, A. Denis and J. Désesquelles \\ Laboratoire de Spectrométrie Ionique et Moléculaire (associé au CNRS), \\ Université Lyon I, Campus de La Doua, 69622 Villeurbanne Cedex, France
}

(Reçu le 25 juillet 1983, accepté le 13 septembre 1983)

\begin{abstract}
Résumé. - Une orientation électronique est produite par effet Stark du second ordre en appliquant un champélectrique sur des niveaux atomiques excités et alignés au préalable par interaction faisceaufeuille. On mesure les fréquences de modulation en alignement et en orientation dans l'hélium en fonction du champ électrique appliqué. On en déduit les polarisabilités tensorielles des niveaux $n^{1} \mathbf{D}$ de l'hélium $(n=3,4,5)$, que l'on compare aux valeurs théoriques.
\end{abstract}

\begin{abstract}
Electronic orientation is produced via second order Stark effect by applying an oblique electric field on excited atomic levels previously aligned by beam-foil interaction. Frequencies of alignment and orientation modulations are measured in helium as a function of the applied electric field strength. The deduced tensor polarizabilities of the ${ }^{4} \mathrm{He}\left(n^{1} \mathrm{D}, n=3,4,5\right)$ levels are compared with theoretical values.
\end{abstract}

Symmetry considerations lead to the possibility of the production of orientation by beamfoil collision when the normal of the foil exit surface does not lie along the ion beam axis [1]. Such orientation of atomic levels has been observed and analysed in a few light species [2] allowing hyperfine quantum beat measurements [3] in both linear- and circular-polarized light. Several authors [4-6] have suggested that the orientation could be caused by the action on the excited level emerging from the foil of an electric field at the exit surface. Lombardi [7] has pointed out the similarity of such a mechanism with the production of orientation by an electric field of a few hundred volts per centimeter observed in atoms excited and aligned by electron bombardment in a discharge cell. In this experiment the orientation is created by a combination of two alignments which are neither parallel nor perpendicular. This symmetry scheme is obtained by the action of an electric field, through second order Stark effect, on an excited atom aligned by electron [8] or ion [9] bombardment, or by linearly polarized light excitation [10].

We describe here a simpler device where the alignment production and the transfer to orientation are decoupled and where the polarization observations do not need any auxiliary magnetic fields as required in Lombardi's experiments. Alignment stems from beam-foil interaction in axial symmetry and transfer to orientation from second order Stark effect by application of an oblique electric field a few centimeters (corresponding to a few nanosecond time of flight) downstream the foil. As an application, precision measurements of tensor polarizabilities of HeI, 1 snd ${ }^{1} \mathrm{D}$ levels with $n=3,4$ and 5 have been achieved using the periodic changes in the linearand circular-polarization components of the emitted light as a function of the field strength. 
A $200 \mathrm{keV}$ energy ${ }^{4} \mathrm{He}^{+}$beam accelerated by a $2 \mathrm{MV}$ Van de Graaff machine is magnetically analysed and directed through a $10(\mp 3) \mu \mathrm{g} / \mathrm{cm}^{2}$ carbon foil. We have verified that, in this axial geometry, the observed light has only a linear polarization component corresponding to the emission from aligned excited particles. Two centimeters down-stream, the beam goes through two conducting parallel plates, biased to $\mp \mathrm{V}$ and $0 \mathrm{~V}$ respectively, which produce a static electric field applied along a particle path of $39.6 \mathrm{~mm}$ at $45^{\circ}$ with respect to the beam axis. An orientation perpendicular to both the electric field direction and the beam axis appears. It is then possible to observe a circular polarization as well as changes in the linear polarization component of the emitted light as a function of the field strength. Figure 1 shows the excitation and detection geometry. Light emitted at $90^{\circ}$ is collected by a lens which directs parallel rays successively through a retarder plate, a linear polarizer and an interference filter. The retarder is a plastic plate rotating around an axis parallel to the light-beam [1]. A second lens focusses the analysed light on a photomultiplier. Photon counts are normalized to total beam charge collected in a Faraday cup.

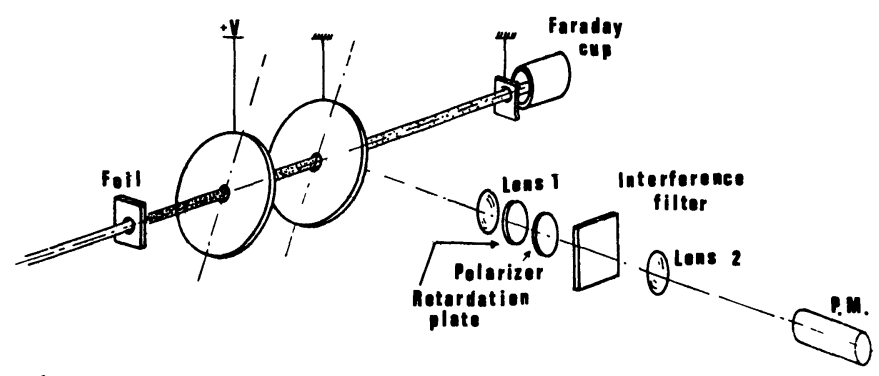

Fig. 1. - Experimental arrangement.

The intensity and polarization of the light are specified by its four Stokes parameters : $I, M$, $C, S$ describing respectively the total intensity, the alignment along the beam direction, the alignment at $45^{\circ}$ with respect to the ion beam axis and the orientation. The intensity of the light observed in given direction and polarization is developed in terms of the density matrix and the detection matrix for an electric dipole. Using the formalism of irreducible tensorial operators, alignment and orientation are expressed for a ${ }^{1} \mathrm{D}_{2}$ level as follows :

$$
\begin{aligned}
& \frac{M}{I}=\frac{\left(-42 Q_{2}+24 Q_{1}+18 Q_{0}\right) \cos 3 \omega t+6\left(Q_{0}-Q_{2}\right) \cos \omega t}{\left(57 Q_{2}+60 Q_{1}+43 Q_{0}\right)-\left(9 Q_{2}-12 Q_{1}+3 Q_{0}\right) \cos 4 \omega t} \\
& \frac{C}{I}=\frac{9 Q_{2}-12 Q_{1}+3 Q_{0}-\left(9 Q_{2}-12 Q_{1}+3 Q_{0}\right) \cos 4 \omega t}{\left(57 Q_{2}+60 Q_{1}+43 Q_{0}\right)-\left(9 Q_{2}-12 Q_{1}+3 Q_{0}\right) \cos 4 \omega t} \\
& \frac{S}{I}=\frac{\left(42 Q_{2}-24 Q_{1}-18 Q_{0}\right) \sin 3 \omega t-18\left(Q_{0}-Q_{1}\right) \sin \omega t}{\left(57 Q_{2}+60 Q_{1}+43 Q_{0}\right)-\left(9 Q_{2}-12 Q_{1}+3 Q_{0}\right) \cos 4 \omega t}
\end{aligned}
$$

$Q_{0}, Q_{1}$ and $Q_{2}$ are the cross sections for excitation of Zeeman sublevels $\left|m_{J}\right|=0,1$ and 2 $\left(Q_{i}=Q_{+i}=Q_{-i}\right) . \omega$ is the electric field splitting between $m_{J}=0$ and $m_{J}= \pm 1$ states $(4 \omega$ between $m=0$ and $m= \pm 2$ states) and $t$ is the transit time of the atoms in the electric field.

The quadratic Stark shift of the state $J, m_{J}$, in a uniform electric field $E$, is the sum of two terms

$$
\Delta W=-\frac{1}{2} \alpha_{\mathrm{sc}}(J) E^{2}-\frac{1}{4} \alpha_{\mathrm{ten}}(J) \frac{3 m_{J}^{2}-J(J+1)}{J(J+1)} 2 E^{2} .
$$

We are concerned here with the tensor part of the polarizability $\alpha_{\text {ten }}$ related to the separation between the $m_{J}=0$ and $m_{J}= \pm 1$ states by the expression $\omega=4 \alpha_{\text {ten }} E^{2}$. The tensor polariza- 
bility can be determined by measurement of the periods of modulation of the relative Stokes parameters by sweeping the electric field strength leaving constant the transit time or alternatively by scanning the observation absciss along the ion beam inside the electric plates at constant voltage. We have used both techniques but here we limit ourselves to the first one. For collecting more light we integrate the emission along a path of more than one centimeter down-stream from the plates in a region where there is no field and consequently no time evolution of the Stokes parameters. The polarizability range attainable by this method is determined by the transit time and the strength of the applied electric field so that the alignment and orientation oscillate at such a velocity to be observable without being averaged out. At $200 \mathrm{keV}$ the transit time is $1.3 \times 10^{-8} \mathrm{~s}$ and with an electric field less than $10 \mathrm{kV} / \mathrm{cm}$ it is possible to measure polarizabilities $\alpha_{\text {ten }}$ which are greater than about $10 \mathrm{~Hz} /(\mathrm{V} / \mathrm{cm})^{2}$. This is the case for the $n^{1} \mathrm{D}$ levels of HeI for $n \geqslant 3$.

Results on the evolution and transfer of alignment and orientation in $\mathrm{HeI}\left(4^{1} \mathrm{D}_{2}\right)$ are displayed in figure 2. Beats in alignment along the beam axis $(M / I)$ and beats in orientation $(S / I)$ are almost in opposite phase because the main frequency of both parameters is due to the $m_{J}=1 \rightarrow m_{J}=2$ transition with pulsation $3 \omega$. The preferred alignment in the electric field direction $(C / I)$ is modulated at the single frequency due to the transition $m_{J}=0 \rightarrow m_{J}=2(4 \omega)$ and is always positive. By Fourier analysis of modulations, only the frequencies corresponding to the ${ }^{1} D_{2}$ level are observed, so that we can neglect the influence of cascades on the obtained result. On figure 2 which reproduces modulations along a complete basic beat period, with an applied field strength growing from zero up to $1.3 \times 10^{5} \mathrm{~V} / \mathrm{m}$, it can be seen that the experimental data are very well fitted by the expressions 1 , with a given set of values for cross sections $Q_{0}$, $Q_{1}$ and $Q_{2}$, so that the theory of second order Stark creation of orientation from combination of two alignments appears well established.

Polarizability of the level $4 d^{1} D_{2}$ has been deduced from the beat frequencies measured in these evolutions of Stokes parameters in HeI $\left(4 d{ }^{1} D_{2}-2 p{ }^{1} P_{1}\right)$ at $4922 \AA$. The uncertainty in the proposed value $\alpha_{\text {ten }}\left(4^{1} \mathrm{D}\right)=0.422(5) \mathrm{kHz} /(\mathrm{V} / \mathrm{cm})^{2}$ is mainly due to the difficulty of exactly mapping the electric field along the ion beam axis. The beam velocity has been measured by the nuclear $(\mathrm{p}, \gamma)$ resonances technique in aluminium. The total systematic error is estimated to about $1 \%$. The standard deviation for eight different measurements is 0.0007 (table I).

Table I. - Tensor polarizability of $n^{1} \mathrm{D}_{2}$ levels in $\mathrm{HeI}\left(\mathrm{kHz} /(\mathrm{V} / \mathrm{cm})^{2}\right)$.

\begin{tabular}{|l|l|l|l|}
\hline$n$ & This work & Szostak et al. $\left({ }^{a}\right)$ & Theory \\
\hline 3 & $0.0145(6)$ & $0.0131(3)$ & $0.014 \cdot 1$ \\
4 & $0.422(5)$ & $0.421(6)$ & 0.4253 \\
5 & $2.63(5)$ & $2.66(6)$ & 2.717 \\
\hline
\end{tabular}

$\left.{ }^{(}{ }^{a}\right)$ D. Szostak, G. Von Oppen and W. D. Perschmann, Ref. [12].

Higher precision is attainable in relative polarizability measured in the $3 d^{1} D_{2}$ and $5 d^{1} D_{2}$ levels at $6678 \AA$ and $4388 \AA$, respectively, with respect to the polarizability of the $4{ }^{1} \mathrm{D}_{2}$ level. In these experiments the modulation curves are recorded at the same electric field, whatever it may be, and also at fixed energy ( $200 \mathrm{keV})$, and consequently at the same velocity of emitting particles, so that systematic errors are considerably reduced. For a shorter experimental time, the evolution of Stokes parameters is recorded alternatively for the two transitions at a high electric field strength only in a small range about zero or an extremum. Corrections are made for taking into account the foil thickening during the measurement (Fig. 3).

Results for polarizabilities in absolute and in relative values are presented in tables I and II. 


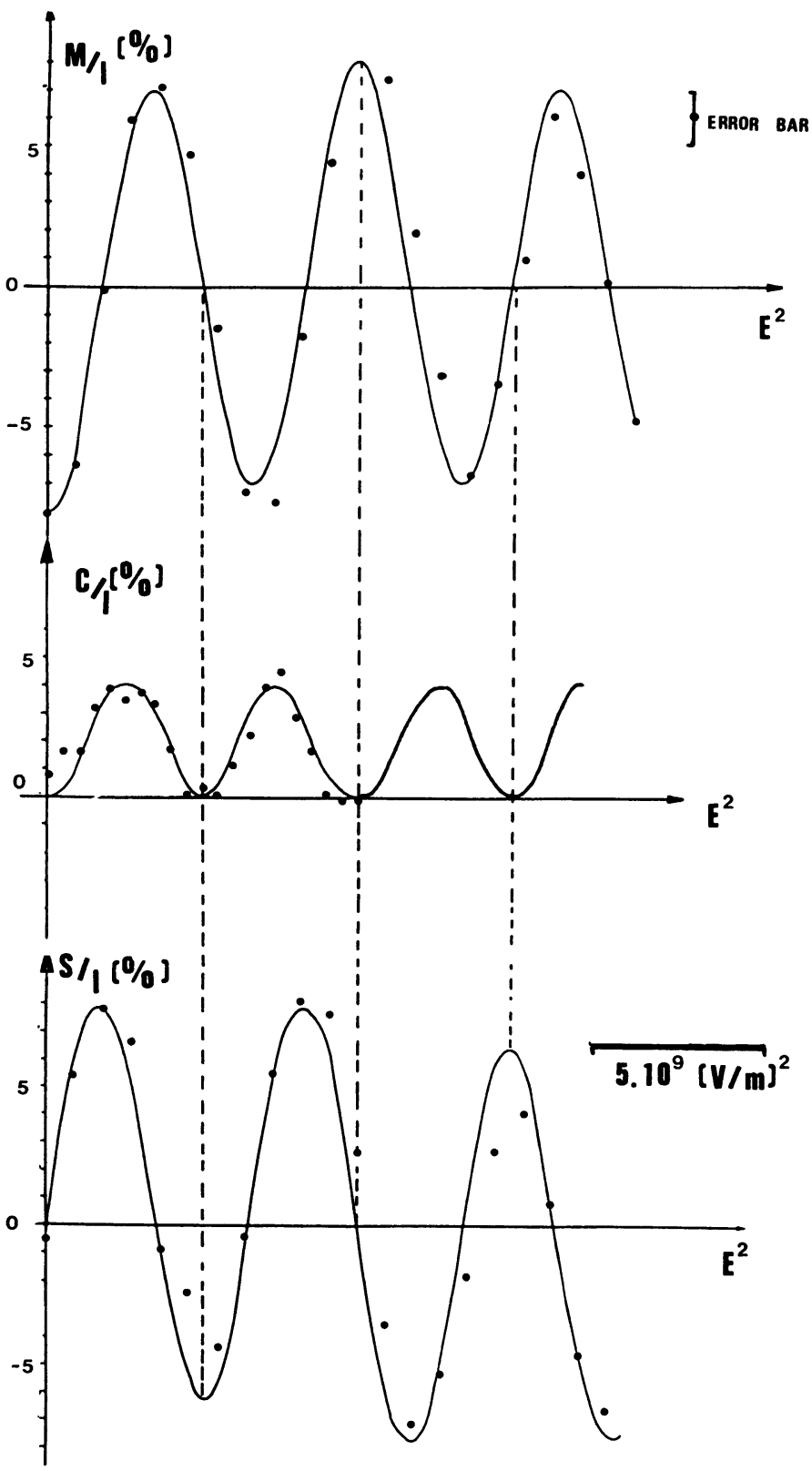

Fig. 2. - Evolution and transfer of alignment and orientation in $\mathrm{HeI}\left(4^{1} \mathrm{D}_{2}\right)$.

Our results are in agreement with the theoretical data obtained in Coulomb approximation within our error bars. The experimental value of Szostak et al. [12] obtained by the level-crossing technique for the $3^{1} \mathrm{D}$ level is widely outside our value, but these authors mentioned to us that they remeasured it and that corrections have to be made to their published result [13].

In résumé, we have shown that our theoretical description of orientation creation from the combination of two alignments and of alignment and orientation transfer by Stark effect is well verified in a beam-foil experiment where the source of the initial alignment and the applied electric field are independent and well separated. Furthermore, we have used the measured modulation 


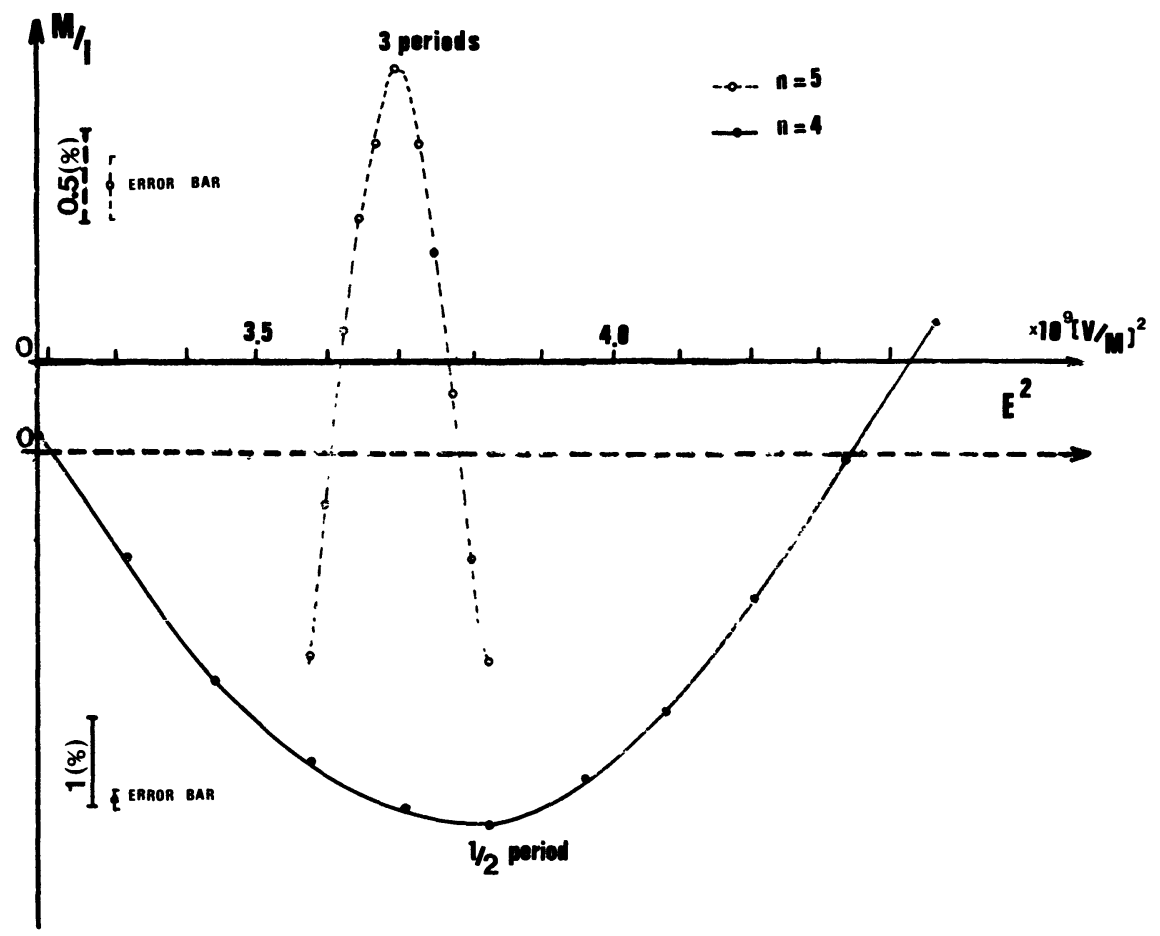

Fig. 3. - Polarizability measurement in $\mathrm{HeI}\left({ }^{1} \mathrm{D}_{2}\right)$ and $\left(5^{1} \mathrm{D}_{2}\right)$. The solid lines are the results of the fits by the theoretical expressions for Stokes parameters.

Table II. - Relative value for tensor polarizability in $\mathrm{HeI}\left(n^{1} \mathrm{D}_{2}\right)$.

\begin{tabular}{|c|c|c|c|}
\hline & This work & Szostak et al. $\left(^{a}\right)$ & Theory \\
\hline$\frac{\alpha_{\text {ten }}\left(4^{1} \mathrm{D}\right)}{\alpha_{\text {ten }}\left(3^{1} \mathrm{D}\right)}$ & $29.03 \pm 0.04\left(^{b}\right)$ & 32.14 & 30.16 \\
$\frac{\alpha_{\text {ten }}\left(5^{1} \mathrm{D}\right)}{\alpha_{\text {ten }}\left(4^{1} \mathrm{D}\right)}$ & $6.22 \pm 0.04\left(^{b}\right)$ & 6.32 & 6.39 \\
\hline
\end{tabular}

$\left({ }^{a}\right)$ D. Szostak, G. Von Oppen and W. D. Perschmann, Ref. [12]. .

(b) Standard deviation.

frequencies of the Stokes parameters to deduce the tensor polarizabilities of $n d{ }^{1} D_{2}$ levels in HeI $(n=3,4,5)$ with a good accuracy. This new method already proves to be simpler and at least as precise as the level-crossing method which is generally used for the determination of the polarizabilities in the range from 10 up to $10^{5} \mathrm{~Hz} /(\mathrm{V} / \mathrm{cm})^{2}$. Work is in progress for improving the precision in absolute measurements of the tensor polarizability using the same quantum beat method. 


\section{References}

[1] FANO, U. and MACEK, J. H., Rev. Mod. Phys. 45 (1973) 553 ;

EluIs, D. G., J. Opt. Soc. Amer 63 (1973) 1232.

[2] Berry, H. G., Curtis, L. J., Ellis, D. G., Schectman, R. M., Phys. Rev. Lett. 32 (1974) 751.

Liu, C. H., Bashin, S., ChurCh, D. A., Phys. Rev. Lett. 33 (1974) 993.

[3] Berry, H. G., Curtis, L. J., Ellis, D. G., Schectman, R. M., Phys. Rev. Lett. 35 (1975) 274.

[4] EcK, T. G., Phys. Rev. Lett. 33 (1974) 1055.

[5] Berry, H. G., Bhardwaj, S. N., Curtis, L. J., Schectman, R. M., Phys. Rev. A 50 (1974) 59

[6] Church, D. A., Kolbe, W., Michel, M. C., Hadeishi, T., Phys. Rev. Lett. 33 (1974) 565.

[7] Lombardi, M., Phys. Rev. Lett. 35 (1975) 1172.

[8] Lombard, M., J. Physique 30 (1969) 631 et Thèse, Université de Grenoble (1969).

[9] Chamoun, E., Lombardi, M., Carré, M., Gaillard, M. L., J. Physique 38 (1977) 591.

[10] Hanle, W., Z. Phys. 35 (1926) 346.

[11] Laloe, F., Ann. Phys. 6 (1971) 5.

[12] Szostak, D., Von Oppen, G. and Perschmann, W. D., Phys. Lett. 76A (1980) 376.

[13] Von OpPen, G., Private communication. 